\title{
OPERATOR KERNELS FOR IRREDUCIBLE REPRESENTATIONS OF EXPONENTIAL LIE GROUPS
}

\author{
DETLEV POGUNTKE
}

(Received 11 February 2008)

\begin{abstract}
A nine-dimensional exponential Lie group $G$ and a linear form $\ell$ on the Lie algebra of $G$ are presented such that for all Pukanszky polarizations $\mathfrak{p}$ at $\ell$ the canonically associated unitary representation $\rho=$ $\rho(\ell, \mathfrak{p})$ of $G$ has the property that $\rho\left(\mathcal{L}^{1}(G)\right)$ does not contain any nonzero operator given by a compactly supported kernel function. This example shows that one of Leptin's results is wrong, and it cannot be repaired.
\end{abstract}

2000 Mathematics subject classification: 43A20, 22E27.

Keywords and phrases: concrete realizations of representations of solvable Lie groups, smooth kernels, polarizations, Beurling algebras.

\section{Introduction}

An extension of Kirillov's original theory for nilpotent Lie groups gives a description of the unitary dual $\widehat{G}$ of an exponential Lie group $G$ in terms of the coadjoint orbits in the linear dual $\mathfrak{g}^{*}$ of the Lie algebra $\mathfrak{g}$ of $G$ - see, for example, [2]: for each $\ell \in \mathfrak{g}^{*}$ one chooses a polarization at $\ell$, that is, a subalgebra $\mathfrak{p}$ which is maximally isotropic with regard to the skew-symmetric form $(X, Y) \longmapsto \ell([X, Y])$ of $\mathfrak{g}$, and which satisfies Pukanszky's condition $P \ell:=\operatorname{Ad}^{*}(P) \ell=\ell+\mathfrak{p}^{\perp}$, where $P=\exp (\mathfrak{p})$. The form $\ell$ defines a character $\chi$ on $P, \chi(\exp X)=e^{i \ell(X)}$, and one may construct $\pi(\ell, \mathfrak{p}):=\operatorname{ind}_{p}^{G} \chi$. This representation is irreducible, its class is independent of the choice of $\mathfrak{p}$ and it is only dependent on the coadjoint orbit from which $\ell$ is taken. In brief, one obtains a bijection from the orbit space $\mathfrak{g}^{*} / G$ onto $\widehat{G}$ (which actually is a homeomorphism, as was shown in [5]).

If $\pi=\pi(\ell, \mathfrak{p})$ then the operators $\pi(f), f \in \mathcal{L}^{1}(G)$, are given by kernels on $G \times G$ satisfying certain covariance conditions with regard to $P \times P$, that is, they are basically functions on $G / P \times G / P$ (using appropriate cross sections). It is a classical task in harmonic analysis to describe the image of the Fourier transform. Here, one may study, for instance, the following problem (for the history of this problem, see also [4]): describe the kernels obtained in that way (or large parts of them). Due

(c) 2008 Australian Mathematical Society 0004-9727/08 \$A2.00+0.00 
to Howe [3], in the nilpotent case, one has a very satisfactory result: all Schwartz functions on $G / P \times G / P$ are in the image of $\mathcal{L}^{1}(G)$, actually obtained by Schwartz functions on $G$. This result was generalized by Ludwig [6] (see also $[5,7,8]$ ) to exponential Lie groups. Ludwig replaced the Schwartz space $\mathcal{S}$ (on $G$ or $G / P$ ) by a modified space $\mathcal{E} \mathcal{S}$ of functions, where $\mathcal{E}$ stands for exponential and means that those functions as well as some of their partial (abelian!) Fourier transforms have an exponential decay in certain directions. In general, the compactly supported smooth functions will not be contained in $\mathcal{E S}$. The latter fact was the motive for Leptin to look for a 'theorem' of the following form.

"TheOREM". If $G$ is an exponential Lie group and $\ell \in \mathfrak{g}^{*}$ then $\pi(\ell, \mathfrak{p})\left(\mathcal{L}^{1}(G)\right)$ contains all operators given by compactly supported smooth kernels [for brevity, we will say that the property $(C K)$ is satisfied], provided that $\mathfrak{p}$ is suitably chosen.

In fact, while Howe and Ludwig's results are true for arbitrary Pukanszky polarizations, an example, given by Leptin [4], shows that the property $(C K)$ depends on the polarization - for this example $(\mathfrak{g}, \ell)$ there exist polarizations at $\ell$ such that $(C K)$ is satisfied, and others where $(C K)$ is violated. It remained a question what 'suitable' might mean in the "theorem". Leptin coined in [4] the notion of a tame polarization, and showed that, for each $\ell \in \mathfrak{g}^{*}$, there exists at least one tame polarization at $\ell$ and 'proved' the following.

THEOREM. If $\mathfrak{p}$ is a tame polarization at $\ell \in \mathfrak{g}^{*}$ then $\pi(\ell, \mathfrak{p})$ has property $(C K)$.

However, Abdennadher and Molitor constructed in [1] a nine-dimensional exponential Lie group $G$, a functional $\ell$ on $\mathfrak{g}$, and a tame polarization $\mathfrak{p}$ at $\ell$ such that $(C K)$ does not hold. Thus, Leptin's 'Theorem' is not true. Two comments on this example may be in order. Firstly, there is a two-dimensional ideal $\mathfrak{a}$ in $\mathfrak{g}$, contained in ker $\ell \cap \mathfrak{g}$ (which implies that $\pi(\ell, \mathfrak{p})$ factors through $G \rightarrow G / \exp \mathfrak{a}$ ), such that the polarization $\mathfrak{p} / \mathfrak{a} \subset \mathfrak{g} / \mathfrak{a}$ of the induced functional is no longer tame. This shows that the notion of tameness is not well behaved under forming quotients, a 'fact' which was used by Leptin. We don't know if this is the only error in [4]. Secondly, in this example, there are other polarizations $\mathfrak{p}^{\prime}$ at $\ell$ such that $(C K)$ holds true.

Hence the original question of a valid "theorem" (with another notion of "suitable polarization') is still open. It is the purpose of this note to present an example of an exponential Lie group $G$ which definitely behaves badly with regard to the above question. There is a form $\ell$ in the linear dual $\mathfrak{g}^{*}$ of $\mathfrak{g}$ such that, for all polarizations $\mathfrak{p}$ at $\ell$, the image $\pi(\ell, \mathfrak{p})\left(\mathcal{L}^{1}(G)\right)$ does not contain a nonzero operator given by a compactly supported kernel. Thus, a revision of the notion of tameness will not help. At the end of the paper we shall present a conjecture, under which conditions one presumably finds all compactly supported smooth kernels in the $\mathcal{L}^{1}$-image.

\section{The Lie algebra}

We start with the five-dimensional Heisenberg algebra $\mathfrak{m}$ with basis $X_{1}, X_{2}, Y_{1}, Y_{2}$, $Z$ and nontrivial brackets

$$
\left[X_{1}, Y_{1}\right]=Z=\left[X_{2}, Y_{2}\right]
$$


To this algebra two further 'nilpotent' elements $V_{1}, V_{2}$ are added with the relations

$$
\left[V_{1}, X_{1}\right]=Y_{1},\left[V_{2}, X_{2}\right]=Y_{2} \text {. }
$$

In that way we obtain a seven-dimensional nilpotent Lie algebra $\mathfrak{n}$ (of step 3) which will be the nilradical of the example algebra $\mathfrak{g}$. The algebra $\mathfrak{n}$ may be thought of as the product of two four-dimensional filiform algebras $\mathcal{L}_{\mathbb{R}}\left(X_{j}, V_{j}, Y_{j}, Z\right), j=1,2$, glued together at the center. The Lie algebra $\mathfrak{g}$ is, as a vector space, the sum of $\mathfrak{n}$ and $\mathcal{L}_{\mathbb{R}}\left(A_{1}, A_{2}\right)$ with additional relations:

$$
\begin{gathered}
{\left[A_{1}, X_{1}\right]=X_{1}, \quad\left[A_{1}, Y_{1}\right]=-Y_{1},} \\
{\left[A_{2}, X_{2}\right]=X_{2}, \quad\left[A_{2}, Y_{2}\right]=-Y_{2},} \\
{\left[A_{1}, V_{1}\right]=-2 V_{1}, \quad\left[A_{2}, V_{2}\right]=-2 V_{2},} \\
\text { and }\left[A_{1}, A_{2}\right]=Z .
\end{gathered}
$$

The functional $\ell \in \mathfrak{g}^{*}$ is just $Z^{*}$, that is, $\ell(Z)=1$, and all other basis elements are in the kernel of $\ell$. One of the features of the pair $\mathfrak{g}, \ell$ is that there are only very few polarizations; in fact, we have the following proposition.

PROPOSITION 2.1. The radical $\mathfrak{g}_{\ell}$ of the skew-symmetric form $(X, Y) \longmapsto \ell([X, Y])$ on $\mathfrak{g}$ is the linear span of $V_{1}, V_{2}, Z$. For each polarization $\mathfrak{p}$ at $\ell$ there are real numbers $\lambda_{1}, \lambda_{2}$ with $\lambda_{1}^{2}+\lambda_{2}^{2}=1$ such that $\mathfrak{p}=\mathcal{L}_{\mathbb{R}}\left(-\lambda_{2} A_{1}+\lambda_{1} A_{2}, V_{1}, V_{2}, Y_{1}, Y_{2}, Z\right)$. In turn, all those spaces are polarizations, and they satisfy Pukanszky's condition.

REMARK 2.2. The linear form $\ell$ is not in general position. Its coadjoint orbit is of dimension six, while there are forms, for instance $Z^{*}+V_{1}^{*}+V_{2}^{*}$, with eightdimensional orbits.

REMARK 2.3. As $\mathfrak{g}_{\ell} \subset \mathfrak{n}$, tameness in the sense of [4] just means that the trace of $\operatorname{ad}(X)$ on $\mathfrak{g} / \mathfrak{p}$ is zero for all $X \in \mathfrak{p}$. This is the case if and only if $\lambda_{1}=\lambda_{2}$.

PROOF. The easy verification of the first sentence is omitted. Let $\mathfrak{p}$ be a given polarization at $\ell$. From the first sentence we conclude

$$
\mathcal{L}_{\mathbb{R}}\left(V_{1}, V_{2}, Z\right) \subset \mathfrak{p}, \quad \operatorname{dim} \mathfrak{p}=6 .
$$

Moreover, as $\mathfrak{g}_{\ell}$ is also the radical of the skew-symmetric form $(X, Y) \longmapsto \ell([X, Y])$ on $\mathfrak{n}$, maximal isotropic subspaces of $\mathfrak{n}$ have to be of dimension 5 . Hence it is impossible that $\mathfrak{p}$ is contained in $\mathfrak{n}$. Therefore, one of the alternatives

$$
\begin{aligned}
& \text { (A) } \operatorname{dim}(\mathfrak{p}+\mathfrak{n})=8, \quad \operatorname{dim} \mathfrak{p} \cap \mathfrak{n}=5, \\
& \text { (B) } \operatorname{dim}(\mathfrak{p}+\mathfrak{n})=9, \quad \operatorname{dim} \mathfrak{p} \cap \mathfrak{n}=4 \text {, }
\end{aligned}
$$

has to hold.

Next we claim

$$
\mathfrak{p} \cap \mathfrak{n} \text { is contained in } \mathcal{L}_{\mathbb{R}}\left(Z, V_{1}, V_{2}, Y_{1}, Y_{2}\right)
$$


If not, by (2.1), there exist coefficients $\alpha_{1}, \alpha_{2}, \beta_{1}, \beta_{2} \in \mathbb{R}$ with $\alpha_{1}^{2}+\alpha_{2}^{2}=1$ such that

$$
W:=\alpha_{1} X_{1}+\alpha_{2} X_{2}+\beta_{1} Y_{1}+\beta_{2} Y_{2} \in \mathfrak{p} \cap \mathfrak{n} .
$$

If both $\alpha_{1}$ and $\alpha_{2}$ are different from zero, we apply $V_{1}$ and $V_{2}$ to $W$, and we conclude that $Y_{1}, Y_{2} \in \mathfrak{p}$. Hence $Z, V_{1}, V_{2}, Y_{1}, Y_{2}, W$ are linearly independent elements of $\mathfrak{n} \cap \mathfrak{p}$, which is impossible. If, say, $\alpha_{2}=0$, but $\alpha_{1} \neq 0$ then $\left[V_{1}, W\right]=\alpha_{1} Y_{1} \in \mathfrak{p}$. But $\left[W, \alpha_{1} Y_{1}\right]=\alpha_{1}^{2} Z=Z$ shows that $\mathfrak{p}$ is not isotropic, and (2.2) is proved. This was the first step to obtain

$$
\mathfrak{p} \cap \mathfrak{n}=\mathcal{L}_{\mathbb{R}}\left(Z, V_{1}, V_{2}, Y_{1}, Y_{2}\right)
$$

Again we argue by contradiction. Suppose that there is strict containment in (2.3). Then only $(B)$ of the alternatives $(A)$ and $(B)$ is possible. Using (2.1), we find coefficients $\alpha_{1}, \ldots, \beta_{2}$ and $\alpha_{1}^{\prime}, \ldots, \beta_{2}^{\prime}$ such that

$$
\begin{aligned}
& W_{1}:=A_{1}+\alpha_{1} X_{1}+\alpha_{2} X_{2}+\beta_{1} Y_{1}+\beta_{2} Y_{2}, \text { and } \\
& W_{2}:=A_{2}+\alpha_{1}^{\prime} X_{1}+\alpha_{2}^{\prime} X_{2}+\beta_{1}^{\prime} Y_{1}+\beta_{2}^{\prime} Y_{2}
\end{aligned}
$$

are in $\mathfrak{p}$. The bracket

$$
\left[W_{1}, W_{2}\right]=\alpha_{1}^{\prime} X_{1}-\alpha_{2} X_{2}-\beta_{1}^{\prime} Y_{1}+\beta_{2} Y_{2}+\left(1+\beta_{1}^{\prime} \alpha_{1}+\alpha_{2} \beta_{2}^{\prime}-\alpha_{1}^{\prime} \beta_{1}-\beta_{2} \alpha_{2}^{\prime}\right) Z
$$

has to be in $\mathfrak{p} \cap \mathfrak{n}$.

If $\alpha_{2} \neq 0$ then $\mathfrak{p} \cap \mathfrak{n} \ni\left[V_{2},\left[W_{1}, W_{2}\right]\right]=-\alpha_{2} Y_{2}$. The relation [[ $\left.\left.W_{1}, W_{2}\right],-\alpha_{2} Y_{2}\right]$ $=\alpha_{2}^{2} Z$ contradicts the isotropy of $\mathfrak{p}$. Hence, we have to have $\alpha_{2}=0$ and, likewise, $\alpha_{1}^{\prime}=0$. In (2.4), the coefficient of $Z$ has to vanish, which, in view of $\alpha_{2}=0=\alpha_{1}^{\prime}$, means that

$$
1+\beta_{1}^{\prime} \alpha_{1}-\beta_{2} \alpha_{2}^{\prime}=0=\alpha_{2}=\alpha_{1}^{\prime} .
$$

Further, $\left[W_{1}, V_{1}\right]=-2 V_{1}-\alpha_{1} Y_{1}$ is in $\mathfrak{p}$. If $\alpha_{1} \neq 0$ then $Y_{1} \in \mathfrak{p}$. But $\ell$ does not vanish on $\left[W_{1}, Y_{1}\right]=-Y_{1}+\alpha_{1} Z$. Hence $\alpha_{1}$ has to be zero, which, using (2.5), implies that $\alpha_{2}^{\prime} \neq 0$. The relation $\left[W_{2}, V_{2}\right]=-2 V_{2}-\alpha_{2}^{\prime} Y_{2}$ shows that $Y_{2}$ is in $\mathfrak{p}$. But $\ell$ does not vanish on the bracket $\left[W_{2}, Y_{2}\right]=-Y_{2}+\alpha_{2}^{\prime} Z$, and this is a contradiction.

Using (2.3) (and $\operatorname{dim} \mathfrak{p}=6$ ), we conclude that there are coefficients $\lambda_{1}, \lambda_{2}, \alpha_{1}, \alpha_{2}$ with $\lambda_{1}^{2}+\lambda_{2}^{2}=1$ such that $\mathfrak{p}$ is spanned by $\mathfrak{p} \cap \mathfrak{n}$ and

$$
W:=-\lambda_{2} A_{1}+\lambda_{1} A_{2}+\alpha_{1} X_{1}+\alpha_{2} X_{2} .
$$

As $\mathfrak{p}$ is isotropic, the linear form $\ell$ has to vanish on the brackets $\left[W, Y_{j}\right]$ for $j=1,2$. But

$$
\left[W, Y_{1}\right] \in \alpha_{1} Z+\mathcal{L}_{\mathbb{R}}\left(Y_{1}, Y_{2}\right) \quad \text { and } \quad\left[W, Y_{2}\right] \in \alpha_{2} Z+\mathcal{L}_{\mathbb{R}}\left(Y_{1}, Y_{2}\right)
$$

show that $\alpha_{1}=0=\alpha_{2}$.

So far we have seen that all polarizations are of the form as claimed in the proposition. The easy verification for the converse fact, namely that each choice of $\lambda_{1}, \lambda_{2}$ yields a polarization satisfying Pukanszky's condition, is omitted. 
For the rest of this article we fix a polarization as in (2.1) and we adapt a basis of $\mathfrak{g}$ accordingly. The basis elements $V_{1}, V_{2}, X_{1}, X_{2}, Y_{1}, Y_{2}, Z$ of $\mathfrak{n}$ remain unchanged, but $A_{1}$ and $A_{2}$ are replaced by $B_{1}:=\lambda_{1} A_{1}+\lambda_{2} A_{2}$ and $B_{2}:=-\lambda_{2} A_{1}+\lambda_{1} A_{2}$ where, of course, $\lambda_{1}^{2}+\lambda_{2}^{2}=1$. In this basis, we have the following, slightly more unpleasant, relations:

$$
\begin{aligned}
& {\left[B_{1}, X_{1}\right]=\lambda_{1} X_{1}, \quad\left[B_{1}, Y_{1}\right]=-\lambda_{1} Y_{1},} \\
& {\left[B_{1}, X_{2}\right]=\lambda_{2} X_{2}, \quad\left[B_{1}, Y_{2}\right]=-\lambda_{2} Y_{2},} \\
& {\left[B_{2}, X_{1}\right]=-\lambda_{2} X_{1}, \quad\left[B_{2}, Y_{1}\right]=\lambda_{2} Y_{1},} \\
& {\left[B_{2}, X_{2}\right]=\lambda_{1} X_{2}, \quad\left[B_{2}, Y_{2}\right]=-\lambda_{1} Y_{2},} \\
& {\left[B_{1}, V_{1}\right]=-2 \lambda_{1} V_{1}, \quad\left[B_{1}, V_{2}\right]=-2 \lambda_{2} V_{2},} \\
& {\left[B_{2}, V_{1}\right]=2 \lambda_{2} V_{1}, \quad\left[B_{2}, V_{2}\right]=-2 \lambda_{1} V_{2},} \\
& {\left[B_{1}, B_{2}\right]=Z, \quad \text { and } \quad \mathfrak{p}=\mathcal{L}_{\mathbb{R}}\left(B_{2}, V_{1}, V_{2}, Y_{1}, Y_{2}, Z\right) .}
\end{aligned}
$$

\section{The group}

Here we present a model for a group with the Lie algebra of the previous section. The Lie algebra $\mathfrak{g}$ is a sum of the ideal $\mathfrak{m}=\mathcal{L}_{\mathbb{R}}\left(X_{1}, X_{2}, Y_{1}, Y_{2}, Z\right)$ and the subalgebra $\mathfrak{u}=\mathcal{L}_{\mathbb{R}}\left(B_{1}, B_{2}, V_{1}, V_{2}, Z\right)$. A particular model for the Heisenberg group, a semidirect product-model, is obtained as follows.

On $M=\mathbb{R}^{5}$ a multiplication is defined by

$$
\begin{aligned}
& \left(x_{1}, x_{2}, y_{1}, y_{2}, z\right)\left(x_{1}^{\prime}, x_{2}^{\prime}, y_{1}^{\prime}, y_{2}^{\prime}, z^{\prime}\right) \\
& \quad=\left(x_{1}+x_{1}^{\prime}, x_{2}+x_{2}^{\prime}, y_{1}+y_{1}^{\prime}, y_{2}+y_{2}^{\prime}, z+z^{\prime}-x_{1} y_{1}-x_{2}^{\prime} y_{2}\right) .
\end{aligned}
$$

For the exponential map exp $: \mathfrak{m} \rightarrow M$ and its inverse $\log : M \rightarrow \mathfrak{m}$ one finds that

$$
\begin{aligned}
& \exp \left(\alpha_{1} X_{1}+\alpha_{2} X_{2}+\beta_{1} Y_{1}+\beta_{2} Y_{2}+\zeta Z\right) \\
& \quad=\left(\alpha_{1}, \alpha_{2}, \beta_{1}, \beta_{2}, \zeta-\frac{1}{2}\left[\alpha_{1} \beta_{1}+\alpha_{2} \beta_{2}\right]\right), \quad \text { and } \\
& \log \left(x_{1}, x_{2}, y_{1}, y_{2}, z\right)=x_{1} X_{1}+x_{2} X_{2}+y_{1} Y_{1}+y_{2} Y_{2} \\
& \quad+\left(z+\frac{1}{2}\left[x_{1} y_{1}+x_{2} y_{2}\right]\right) Z .
\end{aligned}
$$

A realization for a group $U$ with Lie algebra $\mathfrak{u}$ is given as follows.

On $U=\mathbb{R}^{5}$ a multiplication is defined by

$$
\begin{aligned}
& \left(b_{1}, b_{2}, v_{1}, v_{2}, z\right)\left(b_{1}^{\prime}, b_{2}^{\prime}, v_{1}^{\prime}, v_{2}^{\prime}, z^{\prime}\right) \\
& =\left(b_{1}+b_{1}^{\prime}, b_{2}+b_{2}^{\prime}, \exp \left(2 \lambda_{1} b_{1}^{\prime}-2 \lambda_{2} b_{2}^{\prime}\right) v_{1}\right. \\
& \left.\quad+v_{1}^{\prime}, \exp \left(2 \lambda_{2} b_{1}^{\prime}+2 \lambda_{1} b_{2}^{\prime}\right) v_{2}+v_{2}^{\prime}, z+z^{\prime}-b_{1}^{\prime} b_{2}\right) .
\end{aligned}
$$

Using (3.2) and the relations (2.6), we see that elements $\left(b_{1}, b_{2}, 0,0,0\right) \in U$ act on $M$ as automorphisms $I_{b_{1}, b_{2}}$ via

$$
\begin{gathered}
I_{b_{1}, b_{2}}\left(x_{1}, x_{2}, y_{1}, y_{2}, z\right)=\left(x_{1} \exp \left(b_{1} \lambda_{1}-b_{2} \lambda_{2}\right), x_{2} \exp \left(b_{1} \lambda_{2}+b_{2} \lambda_{1}\right),\right. \\
\left.y_{1} \exp \left(-b_{1} \lambda_{1}+b_{2} \lambda_{2}\right), y_{2} \exp \left(-b_{1} \lambda_{2}-b_{2} \lambda_{1}\right), z\right) .
\end{gathered}
$$


Likewise, elements $\left(0,0, v_{1}, v_{2}, 0\right) \in U$ act on $M$ as automorphisms $I_{v_{1}, v_{2}}$ via

$$
I_{v_{1}, v_{2}}\left(x_{1}, x_{2}, y_{1}, y_{2}, z\right)=\left(x_{1}, x_{2}, y_{1}+x_{1} v_{1}, y_{2}+x_{2} v_{2}, z-\frac{1}{2}\left[v_{1} x_{1}^{2}+v_{2} x_{2}^{2}\right]\right) \text {. }
$$

Putting these pieces together, a realization of a group $G$ with Lie algebra $\mathfrak{g}$ is given as follows. On $G=\mathbb{R}^{9}$ a multiplication is defined by

$$
\begin{aligned}
\left(b_{1},\right. & \left.b_{2}, v_{1}, v_{2}, x_{1}, x_{2}, y_{1}, y_{2}, z\right)\left(b_{1}^{\prime}, b_{2}^{\prime}, v_{1}^{\prime}, v_{2}^{\prime}, x_{1}^{\prime}, x_{2}^{\prime}, y_{1}^{\prime}, y_{2}^{\prime}, z^{\prime}\right) \\
= & \left(b_{1}+b_{1}^{\prime}, b_{2}+b_{2}^{\prime}, v_{1} \exp \left(2 \lambda_{1} b_{1}^{\prime}-2 \lambda_{2} b_{2}^{\prime}\right)+v_{1}^{\prime}, v_{2} \exp \left(2 \lambda_{2} b_{1}^{\prime}+2 \lambda_{1} b_{2}^{\prime}\right)\right. \\
& +v_{2}^{\prime}, x_{1} \exp \left(-b_{1}^{\prime} \lambda_{1}+b_{2}^{\prime} \lambda_{2}\right)+x_{1}^{\prime}, x_{2} \exp \left(-b_{1}^{\prime} \lambda_{2}-b_{2}^{\prime} \lambda_{1}\right)+x_{2}^{\prime}, \\
& y_{1} \exp \left(b_{1}^{\prime} \lambda_{1}-b_{2}^{\prime} \lambda_{2}\right)-v_{1}^{\prime} x_{1} \exp \left(-b_{1}^{\prime} \lambda_{1}+b_{2}^{\prime} \lambda_{2}\right)+y_{1}^{\prime}, y_{2} \exp \left(b_{1}^{\prime} \lambda_{2}+b_{2}^{\prime} \lambda_{1}\right) \\
& \quad-v_{2}^{\prime} x_{2} \exp \left(-b_{1}^{\prime} \lambda_{2}-b_{2}^{\prime} \lambda_{1}\right)+y_{2}^{\prime}, z+z^{\prime}-b_{1}^{\prime} b_{2} \\
& +\frac{1}{2}\left[v_{1}^{\prime}, x_{1}^{2} \exp \left(-2 b_{1}^{\prime} \lambda_{1}+2 b_{2}^{\prime} \lambda_{2}\right)+v_{2}^{\prime} x_{2}^{2} \exp \left(-2 b_{1}^{\prime} \lambda_{2}-2 b_{2}^{\prime} \lambda_{1}\right)\right] \\
& -x_{1}^{\prime} y_{1} \exp \left(b_{1}^{\prime} \lambda_{1}-b_{2}^{\prime} \lambda_{2}\right)+v_{1}^{\prime} x_{1}^{\prime} x_{1} \exp \left(-b_{1}^{\prime} \lambda_{1}+b_{2}^{\prime} \lambda_{2}\right) \\
& \left.-x_{2}^{\prime} y_{2} \exp \left(b_{1}^{\prime} \lambda_{2}+b_{2}^{\prime} \lambda_{1}\right)+v_{2}^{\prime} x_{2}^{\prime} x_{2} \exp \left(-b_{1}^{\prime} \lambda_{2}-b_{2}^{\prime} \lambda_{1}\right)\right) .
\end{aligned}
$$

This does not look very pleasant. Fortunately, we shall not really have to work with this multiplication law, but mainly with the inner automorphisms restricted to the normal (Heisenberg) subgroup $M$, which could be derived from the Lie algebra in a more direct manner, that is, without constructing the group $G$. But as we are presenting a counter-example, it seemed to be worthwhile to have the group once written down explicitly. The automorphisms look much simpler, as follows.

If

$$
g=\left(b_{1}, b_{2}, v_{1}, v_{2}, x_{1}, x_{2}, y_{1}, y_{2}, z\right) \in G
$$

and

$$
\left(0,0,0,0, x_{1}^{\prime}, x_{2}^{\prime}, y_{1}^{\prime}, y_{2}^{\prime}, z^{\prime}\right)=:\left[x_{1}^{\prime}, x_{2}^{\prime}, y_{1}^{\prime}, y_{2}^{\prime}, z^{\prime}\right] \in M
$$

then

$$
\begin{aligned}
g\left[x_{1}^{\prime}, x_{2}^{\prime}, y_{1}^{\prime}, y_{2}^{\prime}, z^{\prime}\right] g^{-1}= & {\left[x_{1}^{\prime} \exp \left(b_{1} \lambda_{1}-b_{2} \lambda_{2}\right), x_{2}^{\prime} \exp \left(b_{1} \lambda_{2}+b_{2} \lambda_{1}\right),\left(y_{1}^{\prime}+v_{1} x_{1}^{\prime}\right)\right.} \\
& \times \exp \left(-b_{1} \lambda_{1}+b_{2} \lambda_{2}\right),\left(y_{2}^{\prime}+v_{2} x_{2}^{\prime}\right) \exp \left(-b_{1} \lambda_{2}-b_{2} \lambda_{1}\right), \\
& \left.z^{\prime}-x_{1}^{\prime} y_{1}-x_{2}^{\prime} y_{2}+x_{1} y_{1}^{\prime}+x_{2} y_{2}^{\prime}-\frac{1}{2}\left(v_{1} x_{1}^{\prime 2}+v_{2} x_{2}^{\prime 2}\right)\right] .
\end{aligned}
$$

Introducing appropriate notation the number of variables can be (formally) reduced. On $X:=Y:=\mathbb{R}^{2}$ we have the standard scalar product $\langle\cdot, \cdot\rangle$ and the standard basis. The group $M$ may be written as follows.

$M=X \times Y \times \mathbb{R}$ with multiplication law

$$
(x, y, z)\left(x^{\prime}, y^{\prime}, z^{\prime}\right)=\left(x+x^{\prime}, y+y^{\prime}, z+z^{\prime}-\left\langle x^{\prime}, y\right\rangle\right) .
$$

The reason for introducing different letters $X$ and $Y$ for the same space $\mathbb{R}^{2}$ is that by $x \in X$ we not only mean that $x$ is a vector in $\mathbb{R}^{2}$, but that we think of $x$ as a member of a particular subgroup of $M$. The vector $-x$ is also the inverse of $x$ in $M$, thus one has ' $x^{-1}=-x$ '. 
Notation. Further we define for

$$
\begin{aligned}
g & =\left(b_{1}, b_{2}, v_{1}, v_{2}, x_{1}, x_{2}, y_{1}, y_{2}, z\right) \in G: \delta(g) \\
& =\left(\begin{array}{cc}
\exp \left(b_{1} \lambda_{1}-b_{2} \lambda_{2}\right) & 0 \\
0 & \exp \left(b_{1} \lambda_{2}+b_{2} \lambda_{1}\right)
\end{array}\right) \in G L_{2}(\mathbb{R})=G L(X)=G L(Y),
\end{aligned}
$$

using the standard basis of

$$
\begin{aligned}
\mathbb{R}^{2}, \tau(g) & =-\left(\begin{array}{cc}
v_{1} \exp \left(-b_{1} \lambda_{1}+b_{2} \lambda_{2}\right) & 0 \\
0 & v_{2} \exp \left(-b_{1} \lambda_{2}-b_{2} \lambda_{1}\right)
\end{array}\right) \in M_{2,2}(\mathbb{R}) \\
& =\operatorname{Hom}_{\mathbb{R}}(X, Y), \sigma(g)=\left(\begin{array}{l}
y_{1} \\
y_{2}
\end{array}\right) \in Y
\end{aligned}
$$

and $\omega(g)=\left(\begin{array}{l}x_{1} \\ x_{2}\end{array}\right) \in X$. Note that

$$
\tau(g)=-\left(\begin{array}{cc}
v_{1} & 0 \\
0 & v_{2}
\end{array}\right) \delta(g)^{-1},
$$

and that $\tau\left(g^{-1}\right)=-\tau(g)$.

With these notation the conjugation of $g \in G$ on $[x, y, z] \in M=X \times Y \times \mathbb{R}$ looks as follows.

$$
\begin{aligned}
g[x, y, z] g^{-1}= & {\left[\delta(g) x, \delta(g)^{-1} y-\tau(g) x, z-\langle\sigma(g), x\rangle\right.} \\
& \left.+\langle\omega(g), y\rangle+\frac{1}{2}\langle\delta(g) \tau(g) x, x\rangle\right] .
\end{aligned}
$$

In the following section a prominent role will be played by the subgroup $H:=\{g \in G \mid$ $\omega(g)=0\}$ of $G$. It contains $Y$ as a normal subgroup, and one may form the quotient group $\dot{H}=H / Y$; accordingly we denote by $\dot{h}=h Y$ the coset of $h \in H$ in $\dot{H}$. For $h \in H$ one has

$$
\sigma\left(h^{-1}\right)=-\delta(h)^{-1} \sigma(h) \in Y .
$$

Each element $g \in G$ decomposes uniquely into $g=x h$, where $x \in X, h \in H$. In order to transform $x h$ into $h^{\prime} x^{\prime}\left(h^{\prime} \in H, x^{\prime} \in X\right)$ we define

$$
h_{x}=x h\left(-\delta(h)^{-1} x\right) \quad \text { for } x \in X, h \in H .
$$

More explicitly, we have the following. The element $h_{x}$ is in $H$, and it is also given by the formula

$$
\begin{aligned}
h_{x} & =h[0,-\tau(h) x, \mu(x, h)] \quad \text { with } \\
\mu(x, h) & =-\left\langle\sigma\left(h^{-1}\right), x\right\rangle-\frac{1}{2}\left\langle\delta(h)^{-1} \tau(h)^{-1} x, x\right\rangle \\
& =\left\langle\delta(h)^{-1} \sigma(h), x\right\rangle+\frac{1}{2}\left\langle\delta(h)^{-1} \tau(h) x, x\right\rangle,
\end{aligned}
$$

which can be seen as follows. From (3.10) one gets

$$
h^{-1} x h=\left[\delta\left(h^{-1}\right) x,-\tau\left(h^{-1}\right) x,-\left\langle\sigma\left(h^{-1}\right), x\right\rangle+\frac{1}{2}\left\langle\delta\left(h^{-1}\right) \tau\left(h^{-1}\right) x, x\right\rangle\right] \in M .
$$


Using $\tau\left(h^{-1}\right)=-\tau(h),(3.9)$, one finds according to (3.8) for the product in $M$

$$
h^{-1} x h\left[-\delta(h)^{-1} x, 0,0\right]=\left[0, \tau(h) x,-\left\langle\sigma\left(h^{-1}\right), x\right\rangle-\frac{1}{2}\left\langle\delta(h)^{-1} \tau(h)^{-1} x, x\right\rangle\right],
$$

from which the claim follows; the other form of $\mu$ is obtained by using (3.9) and (3.11).

From (3.13) we conclude, in particular, the following.

$$
\delta\left(h_{x}\right)=\delta(h) \quad \text { for all } h \in H, x \in X .
$$

The coset $\left(h_{x}\right)^{\bullet}$ in $\dot{H}$ differs from $h$ only by the central element $[0,0, \mu(x, h)]$, which is considered as being canonically embedded into $\dot{H}$.

We simply remark that the group $\dot{H}$ is isomorphic to $U$ introduced in (3.3).

\section{The corner $p * \mathcal{L}^{1}(G)_{\bar{\chi}} * p$ of the Banach algebra $\mathcal{L}^{1}(G)_{\bar{\chi}}$}

Recall that we have a unitary character $\chi$ on $\exp \mathfrak{p}$, where $\mathfrak{p}$ is the chosen polarization, see (2.6); in particular, we have $\chi$ on the central subgroup $Z:=\{[0,0, z] \in M \mid z \in \mathbb{R}\}$. Using this restricted character, by a slight abuse of the notation denoted by the same letter $\chi$, we form, as usual, the convolution algebra $\mathcal{L}^{1}(G)_{\bar{\chi}}$ consisting of all measurable functions $f: G \rightarrow \mathbb{C}$ such that $f(g z)$ $=\bar{\chi}(z) f(g)$ for all $z \in Z$ and almost all $g \in G$, and that $|f|$ is integrable with regard to the left Haar measure on $G / Z$. It is the purpose of this section to show that, for suitable idempotents $p$ in the adjoint algebra of $\mathcal{L}^{1}(G)_{\bar{\chi}}$, the corner $p * \mathcal{L}^{1}(G)_{\bar{\chi}} * p$ is isomorphic to a Beurling subalgebra of $\mathcal{L}^{1}(\dot{H})_{\bar{\chi}}$, where the corresponding weight can be computed explicitly. The considerations in this section (and partly in the previous one) are modifications of a part of my earlier paper [10]. Very often we shall identify $G$ with $X \times H$, see (3.12), and the lines in front of it. Under this identification the left Haar measure on $G$ is given by the following.

$$
d(x h)=\Delta(h) d x d h,
$$

where $d x$ is the Lebesgue measure on $X=\mathbb{R}^{2}, d h$ is the left Haar measure on $H$, and $\Delta(h):=\operatorname{det} \delta(h)^{-1}$. This means that the functional $\varphi \longmapsto \int_{\mathbb{R}^{2}} \int_{H} \varphi(x h) \Delta(h) d h d x$ is $G$-left invariant on $\mathcal{C}_{c}(G)$.

This is easily proved using (3.12), (3.13) and (3.14), in particular $\delta\left(h_{x}\right)=\delta(h)$.

For $f \in \mathcal{L}^{1}\left(\mathbb{R}^{2}\right)=\mathcal{L}^{1}(Y)$ the Fourier transform is given by

$$
\widehat{f}(\eta)=\int_{\mathbb{R}^{2}} e^{-i\langle y, \eta\rangle} f(y) d y, \quad \eta \in \mathbb{R}^{2} .
$$

For the moment, take any $u \in \mathcal{S}(Y)=\mathcal{S}\left(\mathbb{R}^{2}\right)=$ Schwartz space on $\mathbb{R}^{2}$ such that $u^{*}=u$, that is, $u(-y)=\overline{u(y)}$ for all $y \in Y$, and that $\|\widehat{u}\|_{2}=1$. Later, we shall specify $u$. For any $f \in \mathcal{L}^{1}(Y), x \in X$, we put

$$
f^{x}(y)=e^{-i\langle x, y\rangle} f(y), y \in Y .
$$


Using this operation we define $p=p_{u}$ on $M$ by

$$
p[x, y, a]=e^{-i z}\left(u^{x} * u\right)(y) .
$$

According to [9], $p$ is contained in $\mathcal{L}^{1}(M)_{\bar{\chi}}$, which is formed analogously to $\mathcal{L}^{1}(G)_{\bar{\chi}}$ and $\mathcal{L}^{1}(\dot{H})_{\bar{\chi}}$. In a canonical fashion, $\mathcal{L}^{1}(M)_{\bar{\chi}}$ is sitting in the adjoint algebra of $\mathcal{L}^{1}(G)_{\bar{\chi}}$. The function $p$ satisfies $p^{*}=p$ and $p * p=p$.

For a function $f \in \mathcal{L}^{1}(\dot{H})_{\bar{\chi}}$, which is continuous and compactly supported modulo $Z$, we define a function $S f$ on $G$ via

$$
(S f)(x h y)=(2 \pi)^{-2} \Delta(h)^{-1 / 2} \int_{X} d x^{\prime} \widehat{u}\left(-x^{\prime}\right) \widehat{u}\left(x-\delta(h) x^{\prime}\right) e^{-i\left\langle x^{\prime}, y\right\rangle} f\left(h_{\delta(h) x^{\prime}}\right),
$$

where $x \in X, y \in Y, h \in H$.

Of course, as $Y$ is contained in $H, S f$ is 'overdetermined', but it is easily checked that $S f$ is well defined. From (3.14), it follows that $f\left(h_{\delta(h) x^{\prime}}^{\bullet}\right)$ $=f\left(h^{\bullet}\left[0,0, \mu\left(\delta(h) x^{\prime}, h\right]\right)\right)$, and using (3.13) yields

$$
f\left(h_{\delta(h) x^{\prime}}^{\bullet}\right)=f\left(h\left[0,0,\left\langle\sigma(h), x^{\prime}\right\rangle+\frac{1}{2}\left\langle\delta(h) \tau(h) x^{\prime}, x^{\prime}\right\rangle\right]\right) .
$$

The covariance property of $f$ then gives the following alternative description of $S f$ :

$$
\begin{aligned}
(S f)(x h y)= & f(h) \Phi(x, h y) \quad \text { with } \Phi(x, h y) \\
= & (2 \pi)^{-2} \Delta(h)^{-1 / 2} \int_{X} d x^{\prime} \widehat{u}\left(-x^{\prime}\right) \widehat{u}\left(x-\delta(h) x^{\prime}\right) e^{-i\left\langle y, x^{\prime}\right\rangle} \\
& \times \exp \left(-i\left\{\left\langle\sigma(h), x^{\prime}\right\rangle+\frac{1}{2}\left\langle\delta(h) \tau(h) x^{\prime}, x^{\prime}\right\rangle\right\}\right) \\
& \quad \text { for } x \in X, y \in Y, h \in H .
\end{aligned}
$$

From this we draw the following consequence. If we define a weight $w=w_{u}$ on $\stackrel{\bullet}{H}=H / Y-$ actually on $H / Y Z-$ by

$$
w(h)=\Delta(h) \int_{X} d x \int_{Y} d y|\Phi(x, h y)|
$$

then $S$ induces an isometric embedding from the Beurling algebra $\mathcal{L}^{1}(\stackrel{\bullet}{H}, w)_{\bar{\chi}}$ into $\mathcal{L}^{1}(G)_{\bar{\chi}}$; we denote this embedding by the same letter $S$.

By direct computation, compare [10], one can show that $S$ is involutive, that is,

$$
S\left(f^{*}\right)=S(f)^{*} \quad \text { for } f \in \mathcal{L}^{1}(\stackrel{\bullet}{H}, w)_{\bar{\chi}} .
$$

In order to show that $S$ is multiplicative (with regard to convolution), and that the image of $S$ is contained in $p * \mathcal{L}^{1}(G)_{\bar{\chi}} * p$, we use representation theory and deduce some formulas, which are also useful for the treatment of the representation $\rho=\operatorname{ind}_{P}^{G} \chi, P=\exp \mathfrak{p}$, which after all is the main subject of this article. Let $\pi^{\prime}$ be any representation of $\dot{H}$ such that $\pi^{\prime}=\chi$ on $Z$. Considering $\pi^{\prime}$ as a representation of $H$, one may form the induced representation $\pi=\operatorname{ind}_{H}^{G} \pi^{\prime}$. Observe that $\rho$ is of that 
type, $\rho=\operatorname{ind}_{H}^{G} \rho^{\prime}$ with $\rho^{\prime}=\operatorname{ind}_{P}^{H} \chi$, so that all that will be said about $\pi$ will apply to $\rho$ as well. We consider the more general representation $\pi$, which may be chosen to be faithful on $\mathcal{L}^{1}(G)_{\bar{\chi}}$ in order to complete our discussion of the properties of the operator $S$. if $\mathfrak{K}$ denotes the representation space of $\pi^{\prime}$ then $\pi$ may be realized in $\mathfrak{H}=\mathfrak{L}^{2}(X, \mathfrak{K})$; actually $\pi$ is given by $\{\pi(x h) \xi\}\left(x^{\prime}\right)=\Delta(h)^{1 / 2} \pi^{\prime}\left(h_{x-x^{\prime}}\right)\left(\xi\left(\delta(h)^{-1}\left(x^{\prime}-x\right)\right)\right) \quad$ for $\xi \in \mathfrak{H}, x^{\prime}, x \in X, h \in H$.

For the orthogonal projection $\pi(p)$, see (4.3), one finds, compare [10]:

$$
\{\pi(p) \xi\}\left(x^{\prime}\right)=\widehat{u}\left(x^{\prime}\right) \int_{X} d x \widehat{u}(x) \xi(x) .
$$

This means in particular that $\pi(p) \mathfrak{H}$ can be canonically identified with $\mathfrak{K}$; the map $V: \mathfrak{K} \rightarrow \pi(p) \mathfrak{H}$, given by

$$
(V \eta)\left(x^{\prime}\right)=\widehat{u}\left(x^{\prime}\right) \eta
$$

is an isometry from $\mathfrak{K}$ onto the range of the projection $\pi(p)$.

Direct computation, see [10], shows that, for any $f \in \mathcal{L}^{1}(\dot{H}, w)_{\bar{\chi}}$, the space $\pi(S f) \mathfrak{H}$ is contained in $\pi(p) \mathfrak{H}$. This implies, as $\pi$ is faithful, that $p * S f=S f$. Since $S$ commutes with the involution we conclude that also $S f * p=S f$. Consequently we get the following.

The range of the isometric map $S: \mathcal{L}^{1}(\stackrel{\bullet}{H}, w)_{\bar{\chi}} \rightarrow \mathcal{L}^{1}(G)_{\bar{\chi}}$ is contained in

$$
p * \mathcal{L}^{1}(G)_{\bar{\chi}} * p .
$$

Again referring to [10], we state that

$$
V^{-1} \pi(S f) V=\pi^{\prime}(f) \text { for all } f \in \mathcal{L}^{1}(\dot{H}, w) \bar{\chi} .
$$

Summing up, we have seen that $S$ is an isometric $*$-morphism from $\mathcal{L}^{1}(\dot{H}, w)_{\bar{\chi}}$ into $p * \mathcal{L}^{1}(G)_{\bar{\chi}} * p$. In order to see that $S$ maps onto $p * \mathcal{L}^{1}(G) \bar{\chi} * p$ take any continuous $F \in \mathcal{L}^{1}(G)_{\bar{\chi}}$ with compact support modulo $Z$, and define $T F$ on $\dot{H}$ by

$$
\begin{aligned}
(T F)(\dot{h})= & \Delta(h)^{3 / 2} \int_{X} d x \int_{X} d x^{\prime} \int_{Y} d y \widehat{u}\left(x^{\prime}\right) \widehat{u}\left(\delta(h)^{-1}\left[x^{\prime}-x\right]\right) \\
& \times \exp \left(i \mu\left(x-x^{\prime}, h y\right)\right) F(x h y) .
\end{aligned}
$$

Obviously, $T F$ is compactly supported modulo $Z$. Along the lines of [10], one can verify that $\pi(p) \pi(F) \pi(p)=\pi(S(T F))$, which shows that $S(T F)=p * F * p$. Therefore, the range of $S$ contains all functions $p * F * p, F$ as above, which form a dense subspace of $p * \mathcal{L}^{1}(G)_{\bar{\chi}} * p$. As the range of the isometric embedding is closed, we conclude that $S$ maps onto $p * \mathcal{L}^{1}(G)_{\bar{\chi}} * p$.

So far we have proved the following proposition.

Proposition 4.14. For any $u \in \mathcal{S}(Y)$ such that $u^{*}=u$ and $\|\widehat{u}\|_{2}=1$ the operator $S$ of (4.4) or (4.5) yields an isometric *-isomorphism from the Banach algebra $\mathcal{L}^{1}(\dot{H}, w)_{\bar{\chi}}$ onto $p * \mathcal{L}^{1}(G)_{\bar{\chi}} * p$, the weight $w$ on $H$ being given by (4.6) and (4.5). 
In order to determine the weight $w$ more explicitly, we specialize $u$ to be a Gaussian function. But first we explain why this will tell us (almost) the weight for general $u$. The reason is that different $u$ 's yield equivalent weights. Choose $u_{0} \in \mathcal{S}(Y)$ so that

$$
\widehat{u}_{0}(x)=C e^{-(1 / 2)\langle x, x\rangle} \quad \text { for } x \in X=\mathbb{R}^{2} \text { with } C=\pi^{-1 / 2} \text {. }
$$

The factor $C$ is necessary for $\left\|\widehat{u}_{0}\right\|_{2}=1$. We retain the notation $p$, $w=w_{u}, S, \Phi$ for a 'general' $u$, while the corresponding objects for $u_{0}$ are denoted by $p_{0}, w_{0}, S_{0}, \Phi_{0}$. The tool for comparing $w$ and $w_{0}$ is the function $k \in \mathcal{L}^{1}(M)_{\bar{\chi}}$ given by $k[x, y, z]=e^{-i z}\left(u_{0}^{x} * u\right)(y)$. It has the following properties.

$$
k^{*}[x, y, z]=e^{-i z}\left(u^{x} * u_{0}\right)(y), \quad k * k^{*}=p_{0}, \quad k^{*} * k=p .
$$

Using $k$ we define

$$
\begin{aligned}
& K: p_{0} * \mathcal{L}^{1}(G)_{\bar{\chi}} * p_{0} \rightarrow p * \mathcal{L}^{1}(G)_{\bar{\chi}} * p \text { and } \\
& K^{\prime}: p * \mathcal{L}^{1}(G)_{\bar{\chi}} * p \rightarrow p_{0} * \mathcal{L}^{1}(G)_{\bar{\chi}} * p_{0} \text { by } \\
& \quad K(F)=k^{*} * F * k \text { and } K^{\prime}(F)=k * F * k^{*},
\end{aligned}
$$

respectively. The equations of (4.16) show that $K$ and $K^{\prime}$ are involutive isomorphisms of Banach algebras (but no longer isometric); in fact, they are inverse to each other. It is a routine matter to verify that the diagram

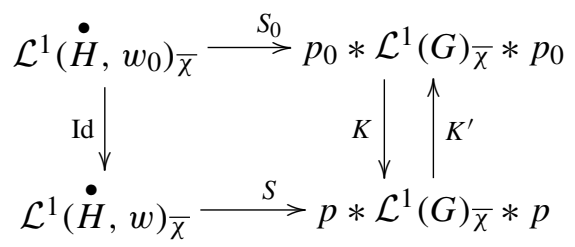

commutes. Consequently, the identity map is a bounded operator from $\mathcal{L}^{1}\left(\stackrel{\bullet}{H}, w_{0}\right)_{\bar{\chi}}$ onto $\mathcal{L}^{1}(\dot{H}, w)_{\bar{\chi}}$, and vice versa. From this fact, we conclude that $w$ and $w_{0}$ are equivalent, that is, there is a positive constant $E$ such that

$$
\frac{1}{E} w(\dot{h}) \leq w_{0}(\dot{h}) \leq E w(\dot{h}) \quad \text { for all } \dot{h} \in \dot{H} .
$$

Now we are going to compute the weight $w_{0}$ explicitly. In order not to interrupt the discussion at a later point, we state here the following formula.

For real numbers $\gamma$ and $v, \gamma$ being positive, one has

$$
\begin{gathered}
\pi^{-1 / 2} \frac{1}{2 \pi} \gamma^{-1 / 2} \int_{-\infty}^{\infty} d x \int_{-\infty}^{\infty} d y \mid \int_{-\infty}^{\infty} d s e^{-(1 / 2) s^{2}} \exp \left(-\frac{1}{2}(x-\gamma s)^{2}\right) \\
\times \exp \left(-i\left\{s y-\frac{1}{2} v s^{2}\right\}\right) \mid=\sqrt{2}\left\{\left(\frac{1}{\gamma}+\gamma\right)^{2}+\left(\frac{v}{\gamma}\right)^{2}\right\}^{1 / 4} .
\end{gathered}
$$


To this end, we recall the well-known formula

$$
\int_{-\infty}^{\infty} d s e^{-(A / 2) s^{2}} e^{-i s z}=\left(\frac{2 \pi}{A}\right)^{1 / 2} \exp \left(-\frac{1}{A} \frac{z^{2}}{2}\right)
$$

for complex numbers $A, z$ with $\Re A>0$.

To evaluate the integral over $s$ in (4.18) we put $A:=1+\gamma^{2}-i v$. For the expression in (4.18) we get

$$
I:=\pi^{-1 / 2} \frac{1}{2 \pi} \gamma^{-1 / 2}\left|\frac{2 \pi}{A}\right|^{1 / 2} \int_{-\infty}^{\infty} d x e^{-x^{2} / 2} \int_{-\infty}^{\infty} d y\left|\exp \left(-\frac{1}{2 A}(y+i x \gamma)^{2}\right)\right| .
$$

As

$$
\begin{aligned}
\left|\exp \left(-\frac{1}{2 A}(y+i x \gamma)^{2}\right)\right|= & \exp \left(\frac{1}{2 A \bar{A}}(x \gamma)^{2}\left(1+\gamma^{2}\right)\right) \\
& \times \exp \left(-\frac{1}{2} \frac{1+\gamma^{2}}{A \bar{A}} y^{2}\right) \exp \left(\frac{v \gamma x y}{A \bar{A}}\right),
\end{aligned}
$$

carrying out the integration over $y$ yields

$$
\begin{aligned}
I= & \pi^{-1 / 2} \frac{1}{2 \pi} \gamma^{-1 / 2}\left|\frac{2 \pi}{A}\right|^{1 / 2}\left(\frac{2 \pi A \bar{A}}{1+\gamma^{2}}\right)^{1 / 2} \\
& \times \int_{-\infty}^{\infty} d x e^{-x^{2} / 2} \exp \left\{\frac{(x \gamma)^{2}\left(1+\gamma^{2}\right)}{2 A \bar{A}}+\frac{(v \gamma x)^{2}}{2\left(1+\gamma^{2}\right) A \bar{A}}\right\} \\
= & \pi^{-1 / 2} \gamma^{-1 / 2}\left(\frac{|A|}{1+\gamma^{2}}\right)^{1 / 2} \int_{-\infty}^{\infty} d x \exp \left(-\frac{1}{2} x^{2} \frac{1}{1+\gamma^{2}}\right) \\
= & \pi^{-1 / 2} \gamma^{-1 / 2}\left(\frac{|A|}{1+\gamma^{2}}\right)^{1 / 2}(2 \pi)^{1 / 2}\left(1+\gamma^{2}\right)^{1 / 2}=\sqrt{2} \gamma^{-1 / 2}|A|^{1 / 2} \\
= & \sqrt{2}\left(\frac{|A|^{2}}{\gamma^{2}}\right)^{1 / 4}=\sqrt{2}\left\{\left(\frac{1}{\gamma}+\gamma\right)^{2}+\left(\frac{v}{\gamma}\right)^{2}\right\}^{1 / 4} .
\end{aligned}
$$

According to (4.5) and (4.6), the weight $w_{0}$ is given by (introducing the new integration variable $y+\sigma(h))$

$$
\begin{aligned}
w_{0}(h)= & \left(\frac{1}{2 \pi}\right)^{2} \Delta(h)^{1 / 2} \int_{X} d x \int_{Y} d y \mid \int_{X} d x^{\prime} \widehat{u}_{0}\left(-x^{\prime}\right) \widehat{u}_{0}\left(x-\delta(h) x^{\prime}\right) \\
& \times \exp \left(-i\left\{\left\langle y, x^{\prime}\right\rangle+\frac{1}{2}\left\langle\delta(h) \tau(h) x^{\prime}, x^{\prime}\right\rangle\right\}\right) \mid
\end{aligned}
$$

Let $h=\left(b_{1}, b_{2}, v_{1}, v_{2}, 0,0,0,0,0\right) \in H$, hence

$$
\delta(h)=\left(\begin{array}{cc}
\gamma_{1} & 0 \\
0 & \gamma_{2}
\end{array}\right)
$$


with $\gamma_{1}=\exp \left(b_{1} \lambda_{1}-b_{2} \lambda_{2}\right)$ and

$$
\gamma_{2}=\exp \left(b_{1} \lambda_{2}+b_{2} \lambda_{1}\right), \quad \delta(h) \tau(h)=-\left(\begin{array}{cc}
v_{1} & 0 \\
0 & v_{2}
\end{array}\right)
$$

and $\Delta(h)=1 /\left(\gamma_{1} \gamma_{2}\right)$, see (3.9) and (4.1). Writing $x=\left(x_{1}, x_{2}\right), y=\left(y_{1}, y_{2}\right)$ and $x^{\prime}=\left(s_{1}, s_{2}\right)$ for the integration variables, we find, plugging in the formula (4.15) for $u_{0}$, that

$$
\begin{aligned}
w_{0}(\dot{h})= & \left(\frac{1}{2 \pi}\right)^{2}\left(\gamma_{1} \gamma_{2}\right)^{-1 / 2} C^{2} \int_{-\infty}^{\infty} \int_{-\infty}^{\infty} d x_{1} d x_{2} \int_{-\infty}^{\infty} \int_{-\infty}^{\infty} d y_{1} d y_{2} \\
& \times \mid \int_{-\infty}^{\infty} \int_{-\infty}^{\infty} d s_{1} d s_{2} \exp \left(-\frac{1}{2}\left(s_{1}^{2}+s_{2}^{2}\right)\right) \\
& \times \exp \left(-\frac{1}{2}\left(x_{1}-\gamma_{1} s_{1}\right)^{2}-\frac{1}{2}\left(x_{2}-\gamma_{2} s_{2}\right)^{2}\right) \\
& \times \exp \left(-i\left\{s_{1} y_{1}+s_{2} y_{2}-\frac{1}{2} v_{1} s_{1}^{2}-\frac{1}{2} v_{2} s_{2}^{2}\right\}\right) \mid .
\end{aligned}
$$

Since $C=\pi^{-1 / 2}$, we readily see that $w_{0}(h)$ is a product of two integrals as considered in (4.18). Hence we find that

$$
w_{0}(h)=2\left\{\left(\frac{1}{\gamma_{1}}+\gamma_{1}\right)^{2}+\left(\frac{v_{1}}{\gamma_{1}}\right)^{2}\right\}^{1 / 4}\left\{\left(\frac{1}{\gamma_{2}}+\gamma_{2}\right)^{2}+\left(\frac{v_{2}}{\gamma_{2}}\right)^{2}\right\}^{1 / 4} .
$$

Summing up we have shown the following proposition.

Proposition 4.20. Let $u \in \mathcal{S}(Y)$ with $u^{*}=u$ and $\|\widehat{u}\|_{2}=1$, and form $p=p_{u}$ according to (4.3). Then the operator $S$, see (4.4) and (4.5), is an involutive isomorphism from $\mathcal{L}^{1}\left(\dot{H}, w_{0}\right)_{\bar{\chi}}$ onto $p * \mathcal{L}^{1}(G)_{\bar{\chi}} * p$, where the weight $w_{0}$ is given by

$$
\begin{aligned}
w_{0}(h)= & 2\left\{\left(\exp \left(-b_{1} \lambda_{1}+b_{2} \lambda_{2}\right)+\exp \left(b_{1} \lambda_{1}-b_{2} \lambda_{2}\right)\right)^{2}\right. \\
& \left.+v_{1}^{2} \exp \left(-2 b_{1} \lambda_{1}+2 b_{2} \lambda_{2}\right)\right\}^{1 / 4}\left\{\left(\exp \left(-b_{1} \lambda_{2}-b_{2} \lambda_{1}\right)\right.\right. \\
& \left.\left.+\exp \left(b_{1} \lambda_{2}+b_{2} \lambda_{1}\right)\right)^{2}+v_{2}^{2} \exp \left(-2 b_{1} \lambda_{2}-2 b_{2} \lambda_{1}\right)\right\}^{1 / 4}
\end{aligned}
$$

if $h=\left(b_{1}, b_{2}, v_{1}, v_{2}, 0,0,0,0,0\right) \in H$.

\section{Finishing the argument}

After these lengthy preparations it is fairly easy to prove the theorem of this article.

THEOREM 5.1. Let $G$ be the group as described in (3.6) with Lie algebra $\mathfrak{g}$, let $\ell=Z^{*} \in \mathfrak{g}^{*}$ be the functional as in the lines before Proposition 2.1, let $\mathfrak{p}$ be any polarization at $\ell$ with associated group $P=\exp (\mathfrak{p})$, and let $\chi \in P^{\wedge}$ be the unitary character corresponding to $\left.\ell\right|_{\mathfrak{p}}$. If $\rho$ denotes the induced representation $\operatorname{ind}_{P}^{G} \chi$ then $\rho\left(\mathcal{L}^{1}(G)\right)$ does not contain a nonzero operator given by a compactly supported kernel. 
PROOF. As we described all polarizations in Section 2, we can, of course, take $\mathfrak{p}$ to be the polarization given in (2.6). We think of $\rho$ as $\rho=\operatorname{ind}_{H}^{G} \rho^{\prime}$ with $\rho^{\prime}=\operatorname{ind}_{P}^{H} \chi$. Recall that $H$ is the subgroup $\left\{\left(b_{1}, b_{2}, v_{1}, v_{2}, 0,0, y_{1}, y_{2}, z\right) \mid b_{1}, b_{2}, v_{1}, v_{2}, y_{1}, y_{2} \in \mathbb{R}\right\}$ of $G$, and that $P$ is its one-codimensional (normal) subgroup consisting of the elements with $b_{1}=0$. Using the most obvious one-parameter subgroup complementary to $P$, the representation $\rho^{\prime}$ is realized in $\mathfrak{K}=\mathcal{L}^{2}(\mathbb{R})$ by

$$
\left(\rho^{\prime}\left(b_{1}, b_{2}, v_{1}, v_{2}, 0,0, y_{1}, y_{2}, z\right) \xi\right)(t)=\exp \left(i\left(z+b_{1} b_{2}-t b_{2}\right)\right) \xi\left(t-b_{1}\right) .
$$

Observe that $\rho^{\prime}$ is trivial on a certain four-dimensional normal subgroup of $H$, that $\left\{\left(b_{1}, b_{2}, z\right)\right\}$ is a Heisenberg group, and that $\rho^{\prime}$ is basically one of the standard models of one of the irreducible representations of the Heisenberg group.

Suppose now that $F \in \mathcal{L}^{1}(G)$ has the property that (the nontrivial) $\rho(F)$ is given by a compactly supported kernel. We may as well assume that $F$ is contained in $\mathcal{L}^{1}(G)_{\bar{\chi}}$ because $\left.\rho\right|_{Z}=\left.\chi\right|_{Z}$. Then $F^{*} * F$ has the same properties. Next we claim the following.

ASSERTION. There exists $u \in \mathcal{S}(Y)$, compare (3.8) and (4.3), such that $u=u^{*}$, $\|\widehat{u}\|_{2}=1, \widehat{u}$ is compactly supported, and with $p=p_{u}$ we have $\rho(p) \rho\left(F^{*} * F\right) \rho(p) \neq$ 0 (and this operator is represented by a compactly supported kernel as well).

Suppose to the contrary that $\rho(p) \rho\left(F^{*} * F\right) \rho(p)=0$ for all $u$ as above. We think of $\rho$ as being realized in $\mathcal{L}^{2}\left(X=\mathbb{R}^{2}, \mathfrak{K}\right)=\mathcal{L}^{2}\left(\mathbb{R}^{2}\right) \otimes \mathcal{L}^{2}(\mathbb{R})=\mathcal{L}^{2}\left(\mathbb{R}^{3}\right)$, compare (4.8). As $\rho(p)$ projects onto $\widehat{u} \otimes \mathfrak{K}$, the equation $\rho(p) \rho\left(F^{*} * F\right) \rho(p)=0$ means that $0=\left\langle\rho\left(F^{*} * F\right) \widehat{u} \otimes \eta, \widehat{u} \otimes \eta\right\rangle=\|\rho(F)(\widehat{u} \otimes \eta)\|^{2}$ for all $\eta \in \mathfrak{K}$. As, clearly, the collection of vectors $\widehat{u} \otimes \eta$ is a total subset of $\mathcal{L}^{2}\left(\mathbb{R}^{3}\right)$, this tells that $\rho(F)$ is zero, which is a contradiction. From the structure of $\rho(p)$ it is immediately clear that $\rho(p) \rho\left(F^{*} * F\right) \rho(p)$ is given by a compactly supported kernel.

From the results of Section 4 , in particular (4.12), we conclude that the operator $\rho\left(p * F^{*} * F * p\right)$ is completely determined by an operator $\rho^{\prime}(f)$ on $\mathfrak{K}$ given by a function $f \in \mathcal{L}^{1}\left(\dot{H}, w_{0}\right)_{\bar{\chi}}$. Also this (nontrivial) operator has to be represented by a compactly supported kernel. We are going to show that this is impossible. Since $\rho^{\prime}$ is trivial on the subgroup of the $v$ 's, to compute $\rho^{\prime}(f)$ we have first to integrate $f$ over those variables. It results a function $f^{\prime}$ on the Heisenberg group $\left\{\left(b_{1}, b_{2}, z \mid\right.\right.$ $\left.b_{1}, b_{2}, z \in \mathbb{R}\right\}$ with a covariance condition, that is, $f^{\prime}\left(b_{1}, b_{2}, z\right)=e^{-i z} f^{\prime \prime}\left(b_{1}, b_{2}\right)$ with some function $f^{\prime \prime}$ on $\mathbb{R}^{2}=\left\{\left(b_{1}, b_{2}\right)\right\}$. The crucial point is that, as $f$ is integrable against the weight $w_{0}$, the function $f^{\prime \prime}$ has at least to be integrable against the weight

$$
\begin{aligned}
w_{0}^{\prime}\left(b_{1}, b_{2}\right):= & 2\left(\exp \left(-b_{1} \lambda_{1}+b_{2} \lambda_{2}\right)+\exp \left(b_{1} \lambda_{1}-b_{2} \lambda_{2}\right)\right)^{1 / 2} \\
& \times\left(\exp \left(-b_{1} \lambda_{2}-b_{2} \lambda_{1}\right)+\exp \left(b_{1} \lambda_{2}+b_{2} \lambda_{1}\right)\right)^{1 / 2}
\end{aligned}
$$

on $\mathbb{R}^{2}$, see (4.20).

From the structure of the representation $\rho^{\prime},(5.2)$, we see that

$$
\left(\rho^{\prime}(f) \xi\right)(t)=\int_{-\infty}^{\infty} K_{f}(t, s) \xi(s) d s
$$


for $\xi \in \mathfrak{K}=\mathcal{L}^{2}(\mathbb{R})$, if the kernel $K_{f}$ on $\mathbb{R}^{2}$ is defined to be

$$
K_{f}(t, s)=\int_{-\infty}^{\infty} d b_{2} f^{\prime \prime}\left(t-s, b_{2}\right)^{-i s b_{2}} .
$$

For almost all $b_{1} \in \mathbb{R}$ the integral $\int_{-\infty}^{\infty} d b_{2}\left|f^{\prime \prime}\left(b_{1}, b_{2}\right)\right| w_{0}\left(b_{1}, b_{2}\right)$ exists. As for any fixed $b_{1}$ the function $b_{2} \longmapsto w_{0}\left(b_{1}, b_{2}\right)$ grows exponentially the Fourier transforms $s \longmapsto \int_{-\infty}^{\infty} d b_{2} f^{\prime \prime}\left(b_{1}, b_{2}\right) e^{-i b_{2} s}$ extend to analytic functions in certain strips depending on $b_{1}$ and the $\lambda$ 's. Therefore, it is impossible that $K_{f}$ is compactly supported (if not zero almost everywhere), and the theorem is proved.

\section{Concluding remarks}

The reader may wonder why we have taken a nine-dimensional counter-example. Why didn't we take, for instance, the following seven-dimensional algebra $\mathfrak{g}_{0}=$ $\mathcal{L}_{\mathbb{R}}\left(A_{1}, A_{2}, X_{1}, X_{2}, Y_{1}, Y_{2}, Z\right)$ with nonvanishing brackets:

$$
\begin{aligned}
{\left[X_{1}, Y_{1}\right] } & =Z=\left[X_{2}, Y_{2}\right], \\
{\left[A_{1}, X_{1}\right.} & =X_{1}, \quad\left[A_{1}, Y_{1}\right]=-Y_{1}, \\
{\left[A_{2}, X_{2}\right] } & =X_{2}, \quad\left[A_{2}, Y_{2}\right]=-Y_{2}, \\
{\left[A_{1}, A_{2}\right] } & =Z ?
\end{aligned}
$$

In fact, if again $\ell=Z^{*}$, then $\mathfrak{p}=\mathcal{L}_{\mathbb{R}}\left(\lambda_{1} A_{2}-\lambda_{2} A_{1}, Y_{1}, Y_{2}, Z\right), \lambda_{1}^{2}+\lambda_{2}^{2}=1$ is a Pukanszky polarization at $\ell$ (which for $\lambda_{1}=\lambda_{2}$ is tame in the sense of [4]). And it can be shown along the lines of this article that the image of $\mathcal{L}^{1}\left(G_{0}\right)$ under ind ${ }_{P}^{G_{0}} \chi(P$ and $\chi$ are formed in the obvious manner) does not contain any operator with compactly supported kernel. The reason for taking $\mathfrak{g}$ is that, besides the above $\mathfrak{p}$ 's, there are more 'strange' polarizations such as

$$
\begin{array}{rlr}
\mathcal{L}_{\mathbb{R}}\left(A_{2}, X_{2}, Y_{1}+\beta X_{1}, Z\right), & \beta \neq 0, & \text { or } \\
\mathcal{L}_{\mathbb{R}}\left(A_{1}+A_{2}, X_{1}+\alpha X_{2}, Y_{2}-\alpha Y_{1}, Z\right), & \alpha \neq 0 . &
\end{array}
$$

These polarizations are less pleasant because they are not invariant under $A_{1}$ and $A_{2}$. We did not investigate these polarizations systematically with regard to compactly supported kernels because we considered it simpler to accept the complication arising from two additional variables (where we had complete control over all the polarizations) than to discuss various types of polarizations separately.

Some further words may be in order as to why Leptin's theorem (and his approach) failed. Leptin refers to the determinant (or trace) on certain homogeneous spaces. But the results of [11] show that not only the determinant counts; rather, all eigenvalues contribute to some sort of 'analytical' structure. In the context of the above mentioned paper the eigenvalues on the stabilizers $G_{f}$ of functionals $f \in \mathfrak{g}^{*}$ are crucial. In the present context, they are less influential because representations on the nilradical can 
be extended against $G_{f}$, but nontrivial eigenvalues on other pieces of polarizations do harm to compactly supported kernels. These sentences may sound a little vague, but they led me to the following rigorous conjecture which presumably is close to being optimal.

Conjecture. Let $G$ be an exponential Lie group with Lie algebra $\mathfrak{g}$; the nilradical $[\mathfrak{g}, \mathfrak{g}]$ of $\mathfrak{g}$ is denoted by $\mathfrak{n}$. Let $f \in \mathfrak{g}^{*}$, and denote by $g$ the restriction of $f$ to $\mathfrak{n}$. The linear form $f$ induces a skew symmetric bilinear form $B=B_{f}$ on $\mathfrak{g}$ by $B(X, Y)=f([X, Y])$; the symbol $\mathfrak{v}^{\perp B}$ for any subspace $\mathfrak{v}$ of $\mathfrak{g}$ denotes the orthogonal space with regard to $B$. The stabilizer algebra $\mathfrak{g}_{g}=\mathfrak{n}^{\perp B}$ of $g$ acts via the adjoint representation on $\mathfrak{n} / \mathfrak{n}_{g}$, where $\mathfrak{n}_{g}=\mathfrak{g}_{g} \cap \mathfrak{n}$. Let $\mathfrak{k} \subset \mathfrak{g}_{g}$ be the common kernel of all eigenvalues of this action of $\mathfrak{g}_{g}$, that is, $\mathfrak{k}$ is the largest subspace of $\mathfrak{g}_{g}$ acting nilpotently on $\mathfrak{n} / \mathfrak{n}_{g}$ or, which is the same, on $\mathfrak{g} / \mathfrak{g}_{f}$. Suppose that one of the following equivalent conditions is satisfied, where $\mathfrak{g}_{f}=$ stabilizer algebra of $f=\mathfrak{g}^{\perp B}$.

(i) $\mathfrak{k}^{\perp B} \subset \mathfrak{g}_{f}+\mathfrak{k}+\mathfrak{n}$,

(ii) $(\mathfrak{k}+\mathfrak{n})^{\perp B} \subset \mathfrak{k}+\mathfrak{g}_{f}+\mathfrak{n}$.

Then there exists a Pukanszky polarization $\mathfrak{p}$ at $f$ such that, with $P=\exp \mathfrak{p}, \chi=\chi_{f} \in$ $P^{\wedge}$, the image of $\mathcal{L}^{1}(G)$ under ind ${ }_{P}^{G} \chi$ contains all operators with compactly supported smooth kernels.

\section{References}

[1] J. Abdennadher and C. Molitor-Braun, 'Operator kernels for irreducible unitary representations of solvable exponential Lie groups', J. Lie Theory 16 (2006), 225-238.

[2] P. Bernat, Représentations des Groupes de Lie Résoluble (Dunod, Paris, 1972).

[3] R. Howe, 'On a connection between nilpotent groups and oscillator integrals associated to singularities', Pacific J. Math. 73 (1977), 329-363.

[4] H. Leptin, 'Irreduzible darstellungen von exponentialgruppen und operatoren mit glatten Kernen', J. Reine Angew. Math. 494 (1998), 1-34.

[5] H. Leptin and J. Ludwig, Unitary Representation Theory of Exponential Lie Groups, Expositions in Mathematics, 18 (de Gruyter, Berlin, 1994).

[6] J. Ludwig, 'Irreducible representations of exponential solvable Lie groups and operators with smooth kernels', J. Reine Angew. Math. 339 (1983), 1-26.

[7] J. Ludwig and C. Molitor-Braun, 'Exponential actions, orbits and their kernels', Bull. Austral. Math. Soc. 57 (1998), 497-513.

[8] C. Molitor-Braun, 'Actions exponentielles et noyaux d'opérateurs' Travaux mathématiques, IX (Centre Universitaire, Luxembourg, 1997), pp. 23-101.

[9] D. Poguntke, 'Gewisse Segalsche Algebren Auf Lokalkompakten Gruppen', Arch. Math. 33 (1979), 454-460.

[10] _ 'Nichtsymmetrische sechsdimensionale Liesche Gruppen', J. Reine Angew. Math. 306 (1979), 154-176.

[11] , Algebraically irreducible representations of $L^{1}$-algebras of exponential Lie groups', Duke Math. J. 50 (1983), 1077-1106.

DETLEV POGUNTKE, Fakultät für Mathematik, Universität Bielefeld, Postfach 100 131, 33501 Bielefeld, Germany

e-mail: poguntke@math.uni-bielefeld.de 